\title{
The Study on Cracking Strength of AIJs to Release the Early-Age Stress of Mass Concrete
}

\author{
Xiaogang Zhang, ${ }^{1}$ Chengqiang Wang, ${ }^{2}$ Shuping Wang, ${ }^{3}$ Feng Xing, ${ }^{1}$ and Ronghua Zhu ${ }^{4}$ \\ ${ }^{1}$ Guangdong Provincial Key Laboratory of Durability for Marine Civil Engineering, College of Civil Engineering, \\ Shenzhen University, Shenzhen 518060, China \\ ${ }^{2}$ Department of Materials and Structural Engineering, Nanjing Hydraulic Research Institute, Nanjing 210024, China \\ ${ }^{3}$ Department of Building Materials, Chalmers University of Technology, 41296 Gothenburg, Sweden \\ ${ }^{4}$ China Ming Yang Wind Power Group Limited, Zhongshan 528437, China \\ Correspondence should be addressed to Xiaogang Zhang; szzxg@szu.edu.cn and Shuping Wang; shuping@chalmers.se
}

Received 18 April 2015; Revised 30 August 2015; Accepted 31 August 2015

Academic Editor: Hossein Moayedi

Copyright (c) 2015 Xiaogang Zhang et al. This is an open access article distributed under the Creative Commons Attribution License, which permits unrestricted use, distribution, and reproduction in any medium, provided the original work is properly cited.

This paper aims to theoretically and numerically assess the effect of setting artificial-induced joints (AIJs) during construction period of a mass concrete structure to release the early-stage thermal stress. With respect to the coupling influences of various factors such as size and boundary of AIJs, an analytical model for its cracking strength on the setting section of mass concrete is proposed based on double-parameter fracture theory. A kind of hyper-finite element analysis (FEA) for many array AIJs in simplified plane pate is also presented by using bilinear cohesive force distribution. The results from the present model and numerical simulation were compared to those of experimental data to prove the efficiency and accuracy of the analytical model and FEA. The model presented in this study for the cracking strength of AIJs provides a simple useful tool to accurately evaluate how many early stress AIJs reduced. The theoretical solution and FEA results could also be significantly contributed to find the "just" and "perfect" release of the temperature stress and to improve the design level of AIJs in mass concrete structure.

\section{Introduction}

In the past decades, mass concrete structures such as super long underground sidewall, large basement slab, and roller compacted concrete (RCC) have been widely used in the practical construction like subway station [1], high-rise building [2], and RCC arch dam [3]. The early irregular cracks caused by the thermal stress of mass concrete during construction period are a worldwide problem to the structure $[4,5]$, and how to effectively control the early-age temperature cracks in the mass concrete structure is significantly concerned by researchers [6-8]. Up to now, many methods have been introduced and applied to eliminate these early random cracks, such as adopting deformation joints [9], setting postpoured strips [2], and using high performance materials [10-13]. Although the above methods to some extent can inhibit the growth of random early-age cracks, there are still problems during the realistic applications $[2,10,14]$.

Recently, therefore, more attention has been paid to a new method of setting artificial-induced joints (AIJs) in construction period to reduce the early-age thermal stress of mass concrete $[1,15,16]$. Figure 1 illustrates the joint treatment by this new method for different types of mass concrete (i.e., super long underground sidewall, large basement slab, and RCC arch dam). As can be seen from Figure 1(c), the cross section of mass concrete consists of many embedded gaps phase-to-phase arrayed if the setting of AIJs in RCC is taken as an example in the present study. The size of each embedded gap is $2 a \times 2 c$; the vertical and horizontal distance between two adjacent embedded gap centres are $2 b$ and $2 d$, respectively. Cross section embedded AIJs are expected to be cracked prior to avoiding random cracks in other parts/sections of the structures when the early temperature rises in mass concrete due to the heat of cement hydration (Figure 1(c)). Then backfill grouting is applied in the cracking section when the chemical reaction of hydration heat has been done during the construction period of mass concrete structures.

The cracking strength of AIJs which is related to the size, the shape of each embedded gap, the distance between two 


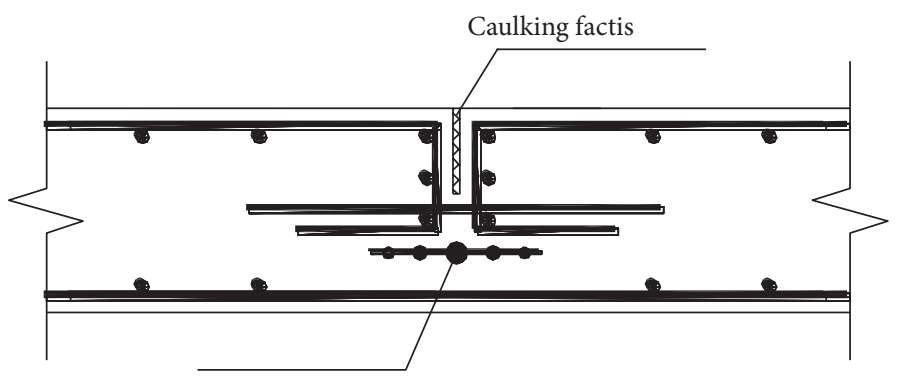

Medium buried rubber waterstop

(a) AIJs in super long underground sidewall

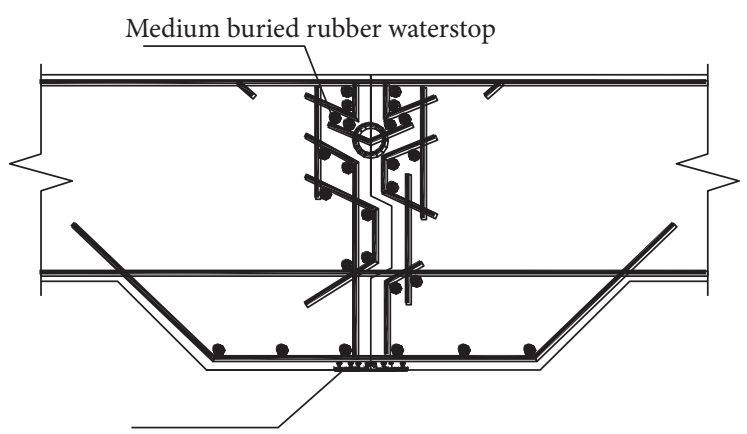

Sticking type of waterstop

(b) AIJs in large basement slab

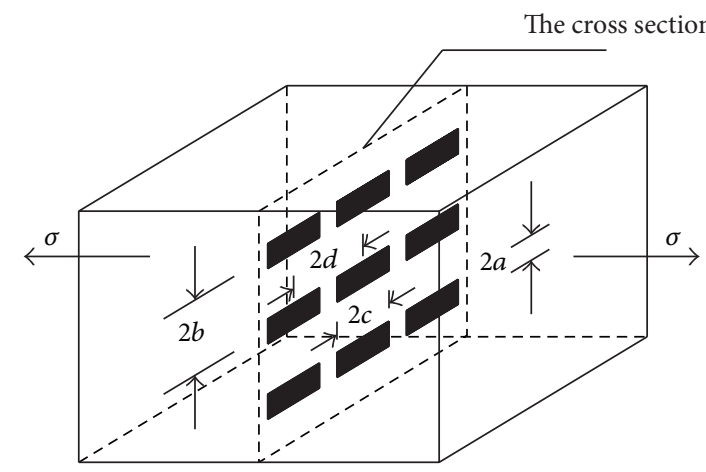

(c) AIJs in RCC

Figure 1: The detailed joint treatments of AIJs for different mass concrete.

cross section embedded AIJs, and so forth is the most key and important parameter in the study of AIJs in mass concrete. This is because only the right value of cracking strength can be expected to avoid random cracks in other parts/sections and thereby to "just" and "perfectly" release temperature stress in mass concrete. If the value of cracking strength is too small or large, the cross section embedded AIJs cannot be cracked firstly or cannot completely reduce the early stress of other parts/sections. In this situation, the setting of AIJs to inhibit the growth of random early-age cracks will to some extent be limited. Therefore it is of great importance to accurately predict the cracking strength of AIJs in evaluating the effect of controlling the early stress in mass concrete structures. Up to now, the accurately analytical solution of cracking strength to "just" and "perfectly" release temperature stress in mass concrete is still not yet available although some experimental investigation, theoretical model, and numerical simulation have been carried out [15-17]. This is because there are so many complex factors such as boundary of cross section, different size, shape of AIJs, concrete strength, and construction schedule which affect the cracking strength $[16,17]$. Therefore, it is necessary and of great importance to illustrate the highly precise model and accurate FEA for the prediction of cracking strength of AIJs so as to be able to evaluate its effect on releasing the early stress [18-20].

In this paper, an analytical model of the cracking strength of AIJs in RCC is studied and improved after considering the various factors such as size and boundary of AIJs. Then a kind of hyper-finite element method along with the experimental

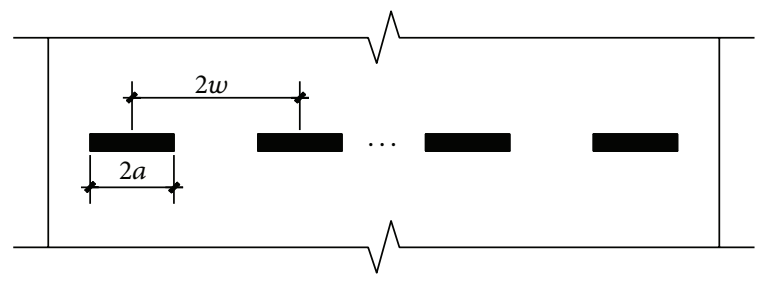

FIgURE 2: Model of penetrated crack in infinite plate.

data is present. The results from the present model and numerical simulation are discussed and further verified. The model presented in this study for the cracking strength is a simple and useful tool to accurately evaluate the reduction of early-age stress. The theoretical solution and FEA results could also be contributed to find the "just" and "perfect" release of the temperature stress and to improve the design level of AIJs in mass concrete structure.

\section{Analytical Model of Cracking Strength for AIJs}

The analytical solution of cracking strength for AIJs in Figure 1(c) can be simplified as a problem of penetrated crack in an infinite plate due to the fact that the length of arch direction is often hundreds of meters (Figure 2). The vertical distance between two adjacent embedded gap centres in Figure 2 is $2 w$. Similar to the development of 
TABLE 1: The indexes for cracking process of AIJ.

\begin{tabular}{lccc}
\hline Fracture energy parameters & Cracking strength parameters & Crack length parameters & Phase of process \\
\hline$G<G_{s}$ & $\sigma<\sigma_{s}$ & $a=a_{0}$ & Before cracking initiation \\
$G_{s} \leq G \leq G_{u}$ & $\sigma_{s} \leq \sigma \leq \sigma_{\max }$ & $a_{0} \leqslant a \leqslant a_{c}$ & Stable growth \\
$G>G_{u}$ & $\sigma>\sigma_{\max }$ & $a>a_{c}$ & Unstable propagation \\
\hline
\end{tabular}

other cracks in concretes, the AIJs also include cracking initiation, stable growth, and unstable propagation process for mass concrete. As assumed, $\sigma$ is the tensile strength along the arch direction, and $\sigma_{s}$ and $\sigma_{\max }$ represent initial and unstable cracking strength, respectively. The propagation of AIJs occurred when thermal stress away from the AIJs is up to $\sigma_{s}$, thereby weakening the cross sections. Similarly, AIJs start to be unstably fractured as thermal stress comes to unstable cracking strength $\sigma_{\max }$. Apparently, one can find that there is a stable propagation stage of the cracking of AIJs between $\sigma_{s}$ and $\sigma_{\max }$.

As mentioned above, the values of $\sigma_{s}$ and $\sigma_{\max }$ are influenced by many factors $[16,17]$. In the present study, the coupling effect of these factors such as boundary of cross section, different size, and concrete strength on the cracking strength of AIJs can be expressed as the following equations $[16,17]$ :

$$
\begin{aligned}
\sigma & =A\left(\alpha_{0}\right) f_{t}\left(1+B\left(\alpha_{0}\right) \frac{a}{a_{\infty}^{*}}\right)^{-1 / 2}, \\
B\left(\alpha_{0}\right) & =\left[\frac{A\left(\alpha_{0}\right)^{Y\left(\alpha_{0}\right)}}{1.12}\right]^{2}, \\
a_{\infty}^{*} & =\frac{E G_{F}}{1.255 \pi f_{t}^{2}},
\end{aligned}
$$

where $\alpha_{0}$ is the ratio of initiation crack length to depth and $G_{F}$ is the fracture energy of concrete materials. $f_{t}$ and $E$ represent the tensile strength and elastic modulus of RCC, respectively. $A\left(\alpha_{0}\right)$ and $Y\left(\alpha_{0}\right)$ are the functions of $\alpha_{0}$ and can be varied from different types of structure.

Although the fracture energy $G_{F}$ in (3) can be generally used to describe the average amount of energy consumed during the breaking down process of AIJs in mass concrete, it cannot effectively express the respective consumption of energy during two important stages of cracking expansion, namely, the stable growth and unstable propagation process [21]. To characterize the energy release ratio in the above different crack expanding periods, $G_{F}$ is divided into two fracture parameters: initiation fracture energy $G_{s}$ and unstable fracture energy $G_{u} \cdot G_{s}$ is associated with cracking initiation stress $\sigma_{s}$ and initial crack length $\alpha_{0}$, while $G_{u}$ is related to maximum stress $\sigma_{\max }$ and critical effective crack length $a_{c}$. More information about the parameters is shown in Table 1.

The solution of $G_{u}$ can be determined by experimental test of the three-point bending beams [21]:

$$
G_{u}=\frac{3 P_{\max }^{2} S^{2}}{4 B^{2} D^{3} E} V^{\prime}(\alpha),
$$

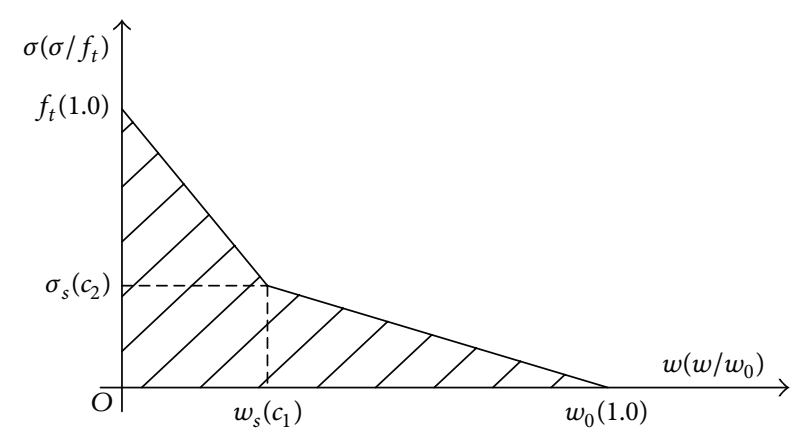

FIGURE 3: Bilinear cohesive force model of mass concrete.

where $B$ and $S$ are the specimen thickness and loading span, respectively. $\alpha=a_{c} / D$ is the ratio of the critical effective crack length to beam depth $D, P_{\max }$ is the maximum load, and $V(\alpha)$ is a coefficient related to $\alpha$ :

$$
\begin{aligned}
& V^{\prime}(\alpha)=\frac{2 \alpha}{(1-\alpha)^{3}}\left[5.58-19.57 \alpha+36.82 \alpha^{2}-34.94 \alpha^{3}\right. \\
& \left.\quad+12.77 \alpha^{4}\right]+\left(\frac{\alpha}{1-\alpha}\right)^{2}(-19.57+73.64 \alpha \\
& \left.\quad-104.82 \alpha^{2}+51.08 \alpha^{3}\right)
\end{aligned}
$$

If the contribution of energy due to cohesive forces is defined as $G_{c}$, the initiation fracture energy $G_{s}$ can also be given as

$$
G_{s}=G_{u}-G_{c}
$$

where $G_{c}$ can be obtained by integration method of cohesive forces on the crack surface.

According to fracture theory, the softening models of concrete [21] can be simplified into the bilinear cohesive force model to describe the cracking behaviors of mass concrete, as illustrated in Figure 3. The expression is

$$
a_{c}=a_{0}+\Delta a_{c}^{*}=a_{0}+\frac{4 r_{0}}{c_{1}+c_{2}}-r_{0} .
$$

In (7), $\Delta a_{c}^{*}$ is the effective crack propagation length, $w$ is the crack opening displacement, and $w_{0}$ is the critical value of $w . r_{0}$ can be regarded as crevice genesis zone, which can be evaluated by the following expression:

$$
r_{0}=\frac{E G_{u}}{2 \pi f_{t}^{2}} .
$$

The stress distribution of the cross section where AIJs embedded will be more affected by the plasticity of concrete 


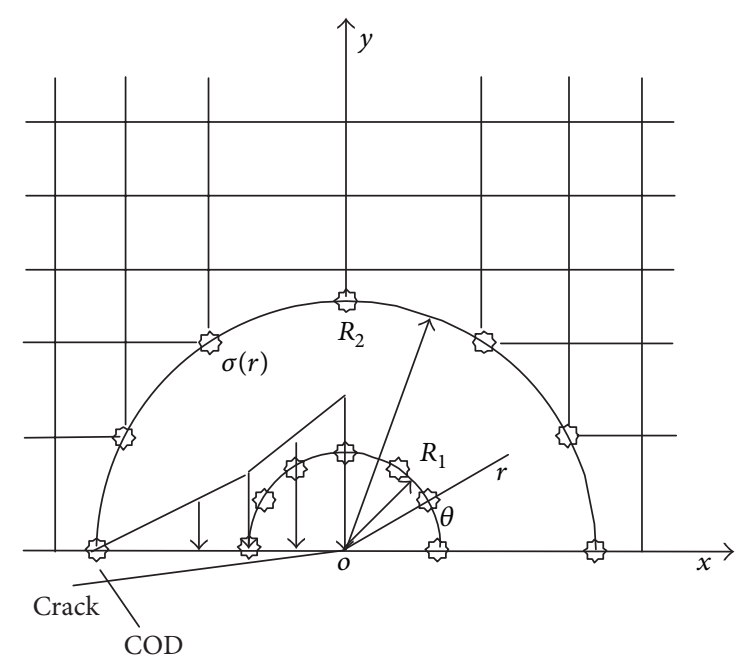

FIGURE 4: Crack-singular-analytical element loaded by the linear cohesive force.

materials with the lager values of the fracture energy of RCC. As a result, the initiation cracking strength $\sigma_{s}$ and the unstable cracking strength $\sigma_{\max }$ of AIJs in mass concrete can be determined by the above values of $G_{s}$ and $G_{u}$ in (4) and (6). The initiation cracking strength $\sigma_{s}$ can be given as

$$
\sigma_{s}=\left(\frac{E G_{s}}{2 W}\right)^{1 / 2}\left[\tan \left(\frac{\pi}{2} \frac{a}{W}\right)\right]^{-1 / 2}
$$

After the stable growth and unstable propagation stage of AIJs, the critical value of unstable cracking strength $\sigma_{\max }$ can also be written as

$$
\sigma_{\max }=\left(\frac{E G_{u}}{2 W}\right)^{1 / 2}\left[\tan \left(\frac{\pi}{2} \frac{a_{c}}{W}\right)\right]^{-1 / 2}
$$

\section{The Hyper-Finite Element Analysis for AIJs}

Among the fracture models of concrete materials proposed by researchers, the fictitious crack model is famous and widely used. It is especially suitable for FEM analysis of AIJs in mass concrete. However, there still exist many difficulties to obtain accurate stress distribution on the vicinity of the crack tip even though a refined mesh is used [21]. An effective way to solve the problem in this study is to apply a hyperelement on the vicinity of AIJs tip based on the Hamiltonian theory of elasticity. Thereafter, the cracking strength of FEM analysis for AIJs in mass concrete is described when the hyperelement is combined with general finite elements.

For bilinear cohesive force distribution in fictitious crack model shown in Figure 4, the propagation process of AIJs in mass concrete can be considered as propagation of elastic cracks, which is a function of a cohesive distribution close force $\sigma(x)$ along the propagation length $\Delta a$. The region of cross section embedded AIJs is dispersed with a ring hyperelement surrounding the crack tip and also ordinary finite elements around the hyperelement, as is illustrated in Figure 4 . The equation of variation in the form of Hamiltonian for the crack-ring-singular element loaded by the linear cohesive force can be expressed as

$$
\begin{aligned}
& \delta\left\{\int_{0}^{\pi} \int_{\ln R_{1}}^{\ln R_{2}}\left\{S_{r} \frac{\partial u}{\partial \xi}+S_{r \theta} \frac{\partial v}{\partial \xi}+\nu S_{r}\left(u+\frac{\partial v}{\partial \theta}\right)+S_{r \theta}\left(\frac{\partial u}{\partial \theta}-v\right)+\frac{E}{2}\left(u+\frac{\partial v}{\partial \theta}\right)^{2}-\frac{1}{2 E}\left[\left(1-v^{2}\right) S_{r}^{2}+2(1+\nu) S_{r \theta}^{2}\right]\right\} d \xi d \theta\right\} \\
& -\int_{\ln R_{1}}^{\ln R_{2}}\left\{\left[k_{3} \exp (2 \xi)+k_{4} \exp (\xi)\right] \cdot v\right\}_{\theta=\pi} d \xi=0,
\end{aligned}
$$

where $u$ and $v$ are the radial and tangential displacement, respectively. $q=\{u, v\}^{T}$ is its displacement vector, $S_{r}=r \sigma_{r}$, $S_{r \theta}=r \tau_{r \theta}$, and $p=\left\{S_{r}, S_{r \theta}\right\}^{T}$ is the dual vector.

The parameters $\zeta, k_{3}, k_{4}$ are obtained by

$$
\begin{aligned}
\xi & =\ln r, \\
k_{3} & =-\frac{\left(\sigma_{s}-\sigma(w)\right)}{\left(R_{2}-R_{1}\right)}, \\
k_{4} & =\frac{\left(\sigma_{s}-\sigma(w) R_{2}\right)}{\left(R_{2}-R_{1}\right)},
\end{aligned}
$$

where $R_{1}=\Delta a_{c}$ and $R_{2}=a-a_{0}$.

Assuming that there are $n_{r}$ nodes on the hyperelement and the unknown variables of each node are displacements of $u, v$ except for the node at $\theta=0$ which has only one variable $u$, so the hyperelement has $2 n_{r}-1$ degrees of freedom. If $\mu_{i}$ is Eigen value and $\psi_{i}=\left\{q_{i}, p_{i}\right\}^{T}$ is Eigen-function vector, the complete state function vector expansion is expressed as

$$
\begin{aligned}
v(\xi, \theta)= & \{q, p\}^{T}=\left\{u, v, S_{r}, S_{r \theta}\right\}^{T} \\
= & c_{\alpha i} \sum_{i=1}^{2 n_{r}-1}\left\{u_{\alpha i}, v_{\alpha i}, S_{r \alpha i}, S_{r \theta \alpha i}\right\}^{T} \\
& +c_{\beta i} \sum_{i=1}^{2 n_{r}-1}\left\{u_{\beta i}, v_{\beta i}, S_{r \beta i}, S_{r \theta \beta i}\right\}^{T}+\widetilde{v}(\xi, \theta) \\
= & c_{\alpha 1} v_{1}^{0}+\sum_{i=1}^{2 n_{r}-2} c_{\alpha i+1} \psi_{\alpha i} \exp \left(\mu_{i} \xi\right)+c_{\beta 1} v_{2}^{0} \\
& +\sum_{i=1}^{2 n_{r}-2} c_{\beta i+1} \psi_{\beta i} \exp \left(-\mu_{i} \xi\right)+\widetilde{v}(\xi, \theta),
\end{aligned}
$$


where $c_{\alpha i}, c_{\beta i}\left(i=1,2, L, 2 n_{r}-1\right)$ are unknown generalized constants and $\widetilde{v}(\xi, \theta)$ is a special solution:

$$
\begin{gathered}
\widetilde{v}(\xi, \theta)=\left\{\widetilde{u}, \widetilde{v}, \widetilde{S}_{r}, \widetilde{S}_{r \theta}\right\}^{T}=k_{4} \exp (\xi)\left\{\frac{1-v}{E}, 0,1,0\right\}^{T} \\
-k_{3} \exp (2 \xi) \\
\cdot\left\{\frac{1-3 v}{6 E} \cos \theta, \frac{5+v}{6 E} \sin \theta, \frac{1}{3} \cos \theta, \frac{1}{3} \sin \theta\right\}^{T} .
\end{gathered}
$$

If $c$ and $d$ are the generalized constant vector and the nodal displacement vector, respectively, the relationship between $c$ and $d$ is

$$
(d-\tilde{d})=T \cdot c, \quad c=T^{-1} \cdot(d-\tilde{d}),
$$

where $c=\left\{c_{\alpha 1}, c_{\alpha 2}, \ldots, c_{\alpha 2 n_{r}-1}, c_{\beta 1}, c_{\beta 2}, \ldots, c_{\beta 2 n_{r}-1}\right\}^{T}, d=$ $\left\{u_{11}, u_{12}, v_{12}, \ldots, u_{1 n_{r}}, v_{1 n_{r}}, u_{21}, u_{22}, v_{22}, \ldots, u_{2 n_{r}}, v_{2 n_{r}}\right\}^{T}, \tilde{d}=$ $\left\{\widetilde{d}_{1}, \widetilde{d}_{2}\right\}^{T}=\left\{\tilde{u}_{11}, \tilde{u}_{12}, \widetilde{v}_{12}, \ldots, \tilde{u}_{1 n_{r}}, \widetilde{v}_{1 n_{r}}, \widetilde{u}_{21}, \widetilde{u}_{22}, \widetilde{v}_{22}, \ldots, \widetilde{u}_{2 n_{r}}\right.$, $\left.\widetilde{v}_{2 n_{r}}\right\}^{T}$, and $\widetilde{d}$ is the value vector of special solution at the nodes.

The components of the inverse of matrix $T$ are

$$
T=\left[\begin{array}{ll}
T_{11} & T_{12} \\
T_{21} & T_{22}
\end{array}\right],
$$

where

$$
\begin{aligned}
\left(T_{11}\right)_{j i} & =\left.u_{\alpha i}\right|_{\theta=\theta_{1}=0} ^{\xi=\ln R_{1}}, \quad j=1, i=1,2, \ldots, 2 n_{r}-1, \\
\left(T_{11}\right)_{j i} & =\left.u_{\alpha i}\right|_{\theta=\theta_{k}} ^{\xi=\ln R_{1}}, \\
j & =2 k-2, k=2,3, \ldots, n_{r}, i=1,2, \ldots, 2 n_{r}-1, \\
\left(T_{11}\right)_{j i} & =\left.v_{\alpha i}\right|_{\theta=\theta_{k}} ^{\xi=\ln R_{1}}, \\
j & =2 k-1, k=2,3, \ldots, n_{r}, i=1,2, \ldots, 2 n_{r}-1, \\
\left(T_{12}\right)_{j i} & =\left.u_{\beta i}\right|_{\theta=\theta_{1}=0} ^{\xi=\ln R_{1}}, \quad j=1, i=1,2, \ldots, 2 n_{r}-1, \\
\left(T_{12}\right)_{j i} & =\left.u_{\beta i}\right|_{\theta=\theta_{k}} ^{\xi=\ln R_{1}}, \\
j & =2 k-2, k=2,3, \ldots, n_{r}, i=1,2, \ldots, 2 n_{r}-1, \\
\left(T_{12}\right)_{j i} & =\left.v_{\beta i}\right|_{\theta=\theta_{k}} ^{\xi=\ln R_{1}}, \\
j & =2 k-1, k=2,3, \ldots, n_{r}, i=1,2, \ldots, 2 n_{r}-1, \\
\left(T_{21}\right)_{j i} & =\left.u_{\alpha i}\right|_{\theta=\theta_{1}=0} ^{\xi=\ln R_{2}}, \quad j=1, i=1,2, \ldots, 2 n_{r}-1, \\
\left(T_{21}\right)_{j i}=\left.u_{\alpha i}\right|_{\theta=\theta_{k}} ^{\xi=\ln R_{2}}, & 2 k-2, k=2,3, \ldots, n_{r}, i=1,2, \ldots, 2 n_{r}-1,
\end{aligned}
$$

$$
\begin{aligned}
\left(T_{21}\right)_{j i} & =\left.v_{\alpha i}\right|_{\theta=\theta_{k}} ^{\xi=\ln R_{2}}, \\
j & =2 k-1, k=2,3, \ldots, n_{r}, i=1,2, \ldots, 2 n_{r}-1, \\
\left(T_{22}\right)_{j i} & =\left.u_{\beta i}\right|_{\theta=\theta_{1}=0} ^{\xi=\ln R_{2}}, \quad j=1, i=1,2, \ldots, 2 n_{r}-1, \\
\left(T_{22}\right)_{j i} & =\left.u_{\beta i}\right|_{\theta=\theta_{k}} ^{\xi=\ln R_{2}}, \\
j & =2 k-2, k=2,3, \ldots, n_{r}, i=1,2, \ldots, 2 n_{r}-1, \\
\left(T_{22}\right)_{j i} & =\left.v_{\beta i}\right|_{\theta=\theta_{k}} ^{\xi=\ln R_{2}}, \\
j & =2 k-1, k=2,3, \ldots, n_{r}, i=1,2, \ldots, 2 n_{r}-1,
\end{aligned}
$$

where $\theta_{k}\left(k=1,2, L n_{r}\right)$ are tangential coordinates of node $\theta$.

The solution can be carried out by iterating over (13) and (11). $R_{0}$ and load vector $F_{0}$ can be obtained by the integration of partial stiffness matrix of hyperelement:

$$
\begin{aligned}
& R_{0}=T^{-T} \cdot R_{c} \bullet T^{-1}, \quad R_{c}=\left[\begin{array}{ll}
R_{11} & R_{12} \\
R_{21} & R_{22}
\end{array}\right], \\
& R_{0}=T^{-T} \bullet\left(F_{c}+R_{c} \bullet T^{-1} \bullet \widetilde{d}\right), \quad F_{c}=\left[F_{1}, F_{2}\right]^{T},
\end{aligned}
$$

where $R_{0}$ and $F_{0}$ are

$$
\begin{aligned}
& \left(R_{11}\right)_{j i}=\int_{0}^{\pi}\left[S_{r \alpha i}(\xi, \theta) u_{\alpha j}(\xi, \theta)\right. \\
& \left.+S_{r \theta \alpha i}(\xi, \theta) v_{\alpha j}(\xi, \theta)\right]\left.\right|_{\xi=\ln R_{1}} ^{\xi=\ln R_{2}} d \theta, \\
& \left(R_{12}\right)_{j i}=\int_{0}^{\pi}\left[S_{r \beta i}(\xi, \theta) u_{\alpha j}(\xi, \theta)\right. \\
& \left.+S_{r \theta \beta i}(\xi, \theta) v_{\alpha j}(\xi, \theta)\right]\left.\right|_{\xi=\ln R_{1}} ^{\xi=\ln R_{2}} d \theta, \\
& \left(R_{21}\right)_{j i}=\int_{0}^{\pi}\left[S_{r \alpha i}(\xi, \theta) u_{\beta j}(\xi, \theta)\right. \\
& \left.+S_{r \theta \alpha i}(\xi, \theta) v_{\beta j}(\xi, \theta)\right]\left.\right|_{\xi=\ln R_{1}} ^{\xi=\ln R_{2}} d \theta, \\
& \left(R_{22}\right)_{j i}=\int_{0}^{\pi}\left[S_{r \beta i}(\xi, \theta) u_{\beta j}(\xi, \theta)\right. \\
& \left.+S_{r \theta \beta i}(\xi, \theta) v_{\beta j}(\xi, \theta)\right]\left.\right|_{\xi=\ln R_{1}} ^{\xi=\ln R_{2}} d \theta, \\
& \left(F_{1}\right)_{j}=-\left.\int_{0}^{\pi}\left[\widetilde{S}_{r} u_{\alpha j}+\widetilde{S}_{r \theta} v_{\alpha j}\right]\right|_{\xi=\ln R_{1}} ^{\xi=\ln R_{2}} d \theta, \\
& \left(F_{2}\right)_{j}=-\left.\int_{0}^{\pi}\left[\widetilde{S}_{r} u_{\beta j}+\widetilde{S}_{r \theta} v_{\beta j}\right]\right|_{\xi=\ln R_{1}} ^{\xi=\ln R_{2}} d \theta \\
& i=1,2, \ldots, 2 n_{r}-1, j=1,2, \ldots, 2 n_{r}-1 .
\end{aligned}
$$


TABLE 2: The FEM and experimental results of unstable fracture energy $G_{u}(\mathrm{~N} / \mathrm{m})$.

\begin{tabular}{lcc}
\hline Specimen designation & Experimental value & FEM \\
\hline A1 & 64.95 & 61.33 \\
A2 & 62.05 & 63.25 \\
A3 & 62.08 & 62.22 \\
\hline B1 & 68.44 & 66.82 \\
B2 & 57.67 & 64.37 \\
B3 & 66.06 & 65.92 \\
\hline
\end{tabular}

\section{Efficiency and Accuracy of the Present Method}

To obtain parameters of fracture energy $\left(G_{s}\right.$ and $\left.G_{u}\right)$ for AIJs of mass concrete, two types of beams with sizes of $800 \times$ $200 \times 100 \mathrm{~mm}$ and $1600 \times 400 \times 200 \mathrm{~mm}$ were prepared for three-point bending measurement. The numerical results and laboratory measurement were then compared to verify the analytical model developed in this study, as well as investigate the accuracy of FEM analysis for prediction. According to the experimental data of RCC and (4)-(5), the values for $G_{s}$ and $G_{u}$ are shown in Figure 5. It can be seen that the evaluated values of $G_{s}$ and $G_{u}$ are independent of both size and boundary. The average values of $G_{s}$ and $G_{u}$ for both series are $15.32 \mathrm{~N} / \mathrm{m}$ and $64.82 \mathrm{~N} / \mathrm{m}$, respectively. And these values are applied to calculate the cracking strength for AIJs in the following discussions.

4.1. FEM Simulation of Three-Point Bending Beams. For the FEM simulation of three-point bending beams, the tangential stress distribution near the crack tip is illustrated in Figure 6. Here, the critical crack length for stable propagation and unstable propagation is $30 \mathrm{~mm}$ and $50 \mathrm{~mm}$, respectively. The numerical results of unstable fracture energy $G_{u}$ for threepoint bending beams, as well as its experimental results, are present in Table 2 . It can be seen that the prediction given by the present FEM is in a good agreement with the experimental data, which demonstrates that the FEM analysis is efficient and accurate for $G_{u}$ prediction.

\subsection{Results of Cracking Strength for AIJs in Mass Concrete.} Whether AIJs in the early period of mass concrete will induce crack or not is important because there is no longer effect if setting of AIJs on the cross section is carried out after thermal stress is released. As a result, the "just" and "perfectly" cracking strength of AIJs in depositing concrete construction period is a key parameter along with the increase of altitude of mass concrete like RCC. Taking into account representative altitude of RCC, the cross section embedded AIJs of mass concrete can be considered as penetrated cracks in infinite plate for the simplification of hyper-finite element analysis (Figure 2). The typical local mesh for artificial-induced joints is shown in Figure 7.

The cracking strength for AIJs in mass concrete predicted by the present model is listed in Table 3. It can be found from Table 3 that the results from FEA are in consistence with those

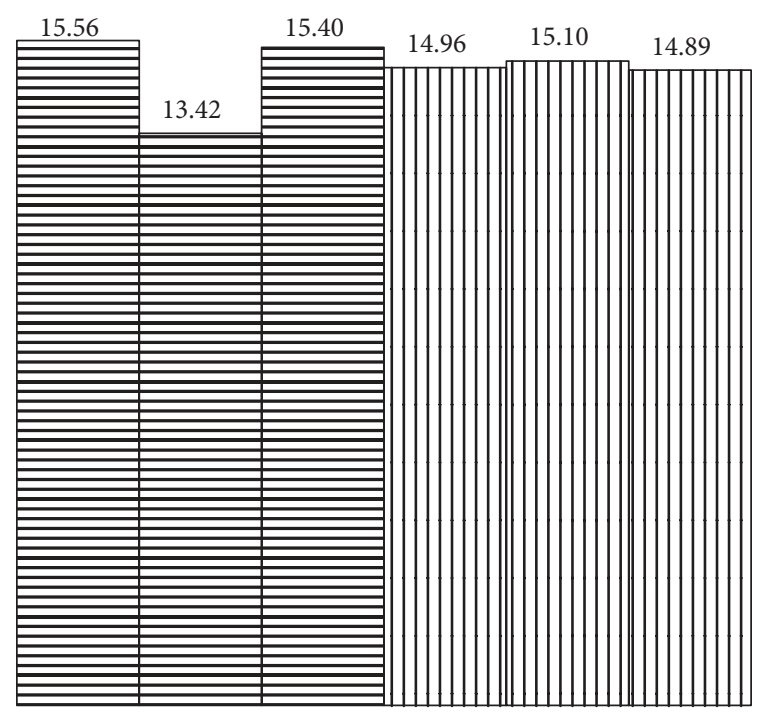

Ш Series A of specimens

$\Xi$ Series B of specimens

(a) Values for $G_{s}(\mathrm{~N} / \mathrm{m})$

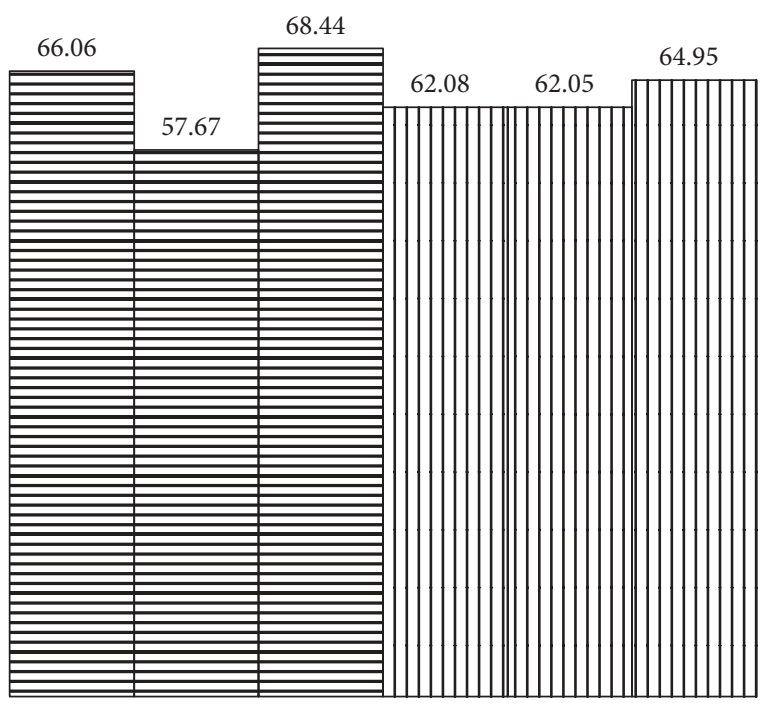

Ш Series A of specimens

曰 Series B of specimens

(b) Values for $G_{u}(\mathrm{~N} / \mathrm{m})$

FIgURE 5: Experimental data for $G_{s}$ and $G_{u}$ of RCC three-point bending beams: series A: $800 \times 200 \times 100 \mathrm{~mm}$; series B: $1600 \times 400 \times$ $200 \mathrm{~mm}$.

from analytical models, indicating that the proposed model can be used to accurately assess the effects of AIJs on releasing the early stress in mass concrete. One can also see when the relative cracking strength of AIJs (i.e., $\sigma_{s} / f_{t}$ and $\sigma_{\max } / f_{t}$ for $2 a=0.3 \mathrm{~m}, 2 b=0.6 \mathrm{~m}$ to $0.9 \mathrm{~m}$ ) reaches about $30 \%-36 \%$ and $55 \%-58 \%$ separately, the AIJs will be initiated to propagate and to be in unstable propagation process, respectively. While for $2 a=0.6 \mathrm{~m}, 2 b=1.2 \mathrm{~m}$ to $1.5 \mathrm{~m}$, the above two values of relative cracking strength slightly decrease to about $23 \%-28 \%$ 
TABLE 3: Cracking strength for AIJs in mass concrete.

\begin{tabular}{|c|c|c|c|c|}
\hline \multirow{3}{*}{ Cracking strength } & Analytical model & FEM & Analytical $n$ & FEM \\
\hline & \multicolumn{4}{|c|}{$2 a=0.3 \mathrm{~m}$} \\
\hline & \multicolumn{2}{|c|}{$2 b=0.6 \mathrm{~m}$} & \multicolumn{2}{|c|}{$2 b=0.9 \mathrm{~m}$} \\
\hline$\sigma_{\max }$ & 1.294 & 1.339 & 1.374 & 1.365 \\
\hline$\sigma_{\max } / f_{t}$ & 0.552 & 0.571 & 0.586 & 0.582 \\
\hline$\sigma_{s}$ & 0.788 & 0.693 & 0.837 & 0.851 \\
\hline$\sigma_{s} / f_{t}$ & 0.336 & 0.296 & 0.357 & 0.363 \\
\hline \multirow{2}{*}{ Cracking strength } & \multicolumn{4}{|c|}{$2 a=0.6 \mathrm{~m}$} \\
\hline & \multicolumn{2}{|c|}{$2 b=1.2 \mathrm{~m}$} & \multicolumn{2}{|c|}{$2 b=1.5 \mathrm{~m}$} \\
\hline$\sigma_{\max }$ & 1.102 & 1.293 & 1.113 & 1.019 \\
\hline$\sigma_{\max } / f_{t}$ & 0.470 & 0.551 & 0.475 & 0.435 \\
\hline$\sigma_{s}$ & 0.557 & 0.612 & 0.578 & 0.658 \\
\hline$\sigma_{s} / f_{t}$ & 0.237 & 0.261 & 0.247 & 0.281 \\
\hline
\end{tabular}

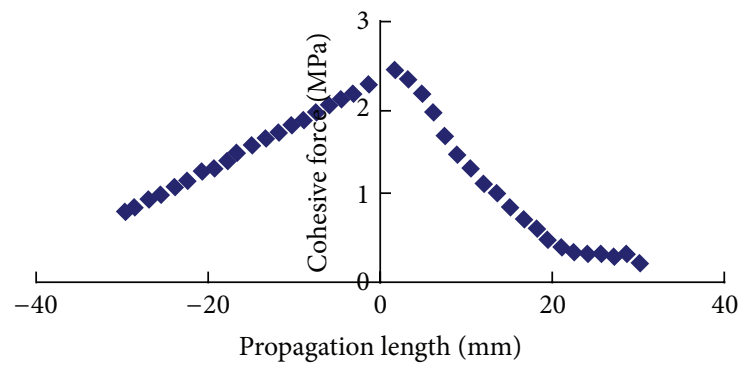

(a)

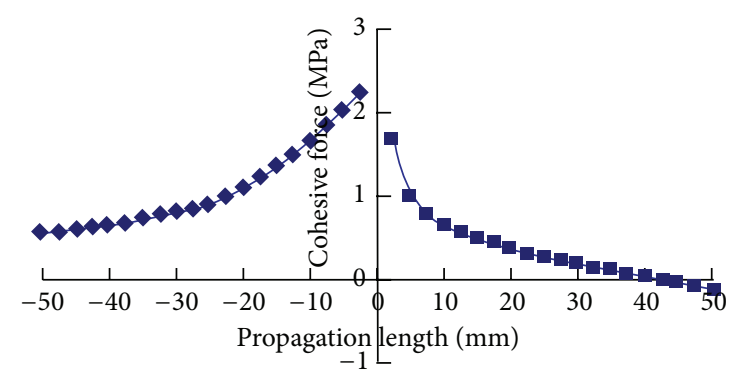

(b)

FIGURE 6: Tangential stress distribution near the crack tip: (a) the subcritical point under maximum load and (b) an unstable propagation point.

and $43 \%-55 \%$. These results can be considered as the basic findings to assess the reduction of early stress of AIJs in mass concrete structure.

\section{Conclusions}

The effect of setting artificial-induced joints (AIJs) during the construction period of mass concrete structures to release the early-stage thermal stress is theoretically and numerically assessed in this study. An analytical model which is related to the cracking strength of AIJs on the setting section of mass concrete, along with its hyper-finite element analysis in simplified plane pate, is also proposed based on the consideration on coupling influence of various factors such

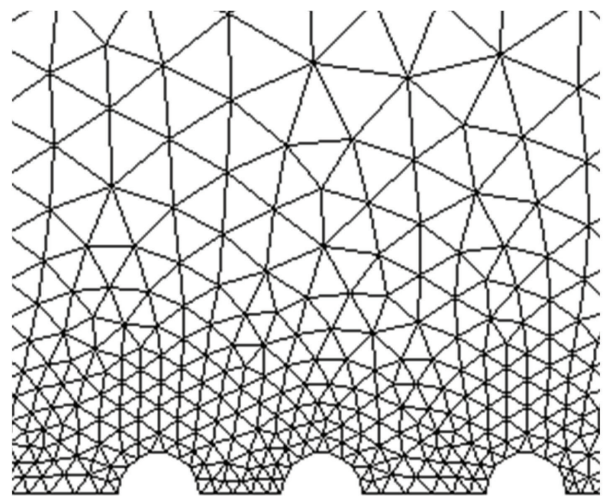

FIGURE 7: Local mesh of hyper-FEM for AIJs (number of hyperelement: 41, radius: $0.175 \mathrm{~m}$, nodes: 4420 , and elements: 8138 ).

as size and boundary effect. The numerical results, together with the experimental data, prove that the analytical model suggested in this study is efficient and accurate in assessing the cracking strength of AIJs. The basic findings can also be used to describe the effects of AIJs on "just" and "perfect" releasing the early-age stress as well as further improve the design level of AIJs in the mass concrete.

\section{Conflict of Interests}

The authors declare that there is no conflict of interests regarding the publication of this paper.

\section{Acknowledgments}

This research was supported by the National Natural Science Foundation of China (51278304), the National Basic Research Program (973 Program) of China (2011CB013604), and the Technology Research and Development Program (basic research project) of Shenzhen, China (JCYJ20120613174456685 and JCYJ20130329143859418). The authors greatly appreciate these supports. 


\section{References}

[1] X. G. Zhang, Y. P. Song, and Z. M. Wu, "Calculation model of equivalent strength for induced crack based on double- $K$ fracture theory and its optimizing setting in RCC arch dam," Transactions of Tianjin University, vol. 1, pp. 8-15, 2005.

[2] J.-H. Ha, Y. S. Jung, and Y.-G. Cho, "Thermal crack control in mass concrete structure using an automated curing system," Automation in Construction, vol. 45, pp. 16-24, 2014.

[3] K. D. Hansen, "Roller compacted concrete: a civil engineering innovation concrete," International Design \& Construction, vol. 18, no. 3, pp. 25-31, 1996.

[4] Y. Yuan and Z. L. Wan, "Prediction of cracking within early-age concrete due to thermal, drying and creep behavior," Cement and Concrete Research, vol. 32, no. 7, pp. 1053-1059, 2002.

[5] N. Liu and G.-T. Liu, "Time-dependent reliability assessment for mass concrete structures," Structural Safety, vol. 21, no. 1, pp. 23-43, 1999.

[6] S. H. Chen, P. F. Su, and I. Shahrour, "Composite element algorithm for the thermal analysis of mass concrete: simulation of lift joint," Finite Elements in Analysis and Design, vol. 47, no. 5, pp. 536-542, 2011.

[7] Y. Wu and R. Luna, "Numerical implementation of temperature and creep in mass concrete," Finite Elements in Analysis and Design, vol. 37, no. 2, pp. 97-106, 2001.

[8] Z. Yunchuana, B. Liangb, Y. Shengyuana, and C. Gutinga, "Simulation analysis of mass concrete temperature field," Procedia Earth and Planetary Science, vol. 5, pp. 5-12, 2012.

[9] J. Yang, Y. Hu, Z. Zuo, F. Jin, and Q. Li, "Thermal analysis of mass concrete embedded with double-layer staggered heterogeneous cooling water pipes," Applied Thermal Engineering, vol. 35, no. 1, pp. 145-156, 2012.

[10] B. I. Yoshitake, H. Wong, T. Ishida, and A. Y. Nassif, “Thermal stress of high volume fly-ash (HVFA) concrete made with limestone aggregate," Construction and Building Materials, vol. 71, pp. 216-225, 2014.

[11] O. Kayali and M. Sharfuddin Ahmed, "Assessment of high volume replacement fly ash concrete-concept of performance index," Construction and Building Materials, vol. 39, pp. 71-76, 2013.

[12] D. Jeon, Y. Jun, Y. Jeong, and J. E. Oh, "Microstructural and strength improvements through the use of $\mathrm{Na}_{2} \mathrm{CO}_{3}$ in a cementless $\mathrm{Ca}(\mathrm{OH})_{2}$-activated class $\mathrm{F}$ fly ash system," Cement and Concrete Research, vol. 67, pp. 215-225, 2015.

[13] J. Tikkanen, A. Cwirzen, and V. Penttala, "Effects of mineral powders on hydration process and hydration products in normal strength concrete," Construction and Building Materials, vol. 72, pp. 7-14, 2014.

[14] F. Lo Monte and P. G. Gambarova, "Thermo-mechanical behavior of baritic concrete exposed to high temperature," Cement \& Concrete Composites, vol. 53, pp. 305-315, 2014.

[15] X.-Z. Wang, Y.-P. Song, J.-K. Dong, and Z.-Q. Huang, "Study on equivalent strength for crack directors of RCC arch dam based on size effect rule," Journal of Harbin Institute of Technology, vol. 16, no. 2, pp. 149-153, 2009.

[16] K. Duan, X. Z. Hu, and F. H. Wittmann, "Boundary effect on concrete fracture and non-constant fracture energy distribution," Engineering Fracture Mechanics, vol. 70, no. 16, pp. 22572268, 2003.

[17] K. Duan, X.-Z. Hu, and F. H. Wittmann, "Size effect on fracture resistance and fracture energy of concrete," Materials and Structures, vol. 36, no. 256, pp. 73-80, 2003.
[18] Y. Ballim, "A numerical model and associated calorimeter for predicting temperature profiles in mass concrete," Cement \& Concrete Composites, vol. 26, no. 6, pp. 695-703, 2004.

[19] Y. Li, L. Nie, and B. Wang, "A numerical simulation of the temperature cracking propagation process when pouring mass concrete," Automation in Construction, vol. 37, pp. 203-210, 2014.

[20] I. Chu, Y. Lee, M. N. Amin, B.-S. Jang, and J.-K. Kim, "Application of a thermal stress device for the prediction of stresses due to hydration heat in mass concrete structure," Construction and Building Materials, vol. 45, pp. 192-198, 2013.

[21] H. W. Reinhardt and S. L. Xu, "Crack extension resistance based on the cohesive force in concrete," Engineering Fracture Mechanics, vol. 64, no. 5, pp. 563-587, 1999. 

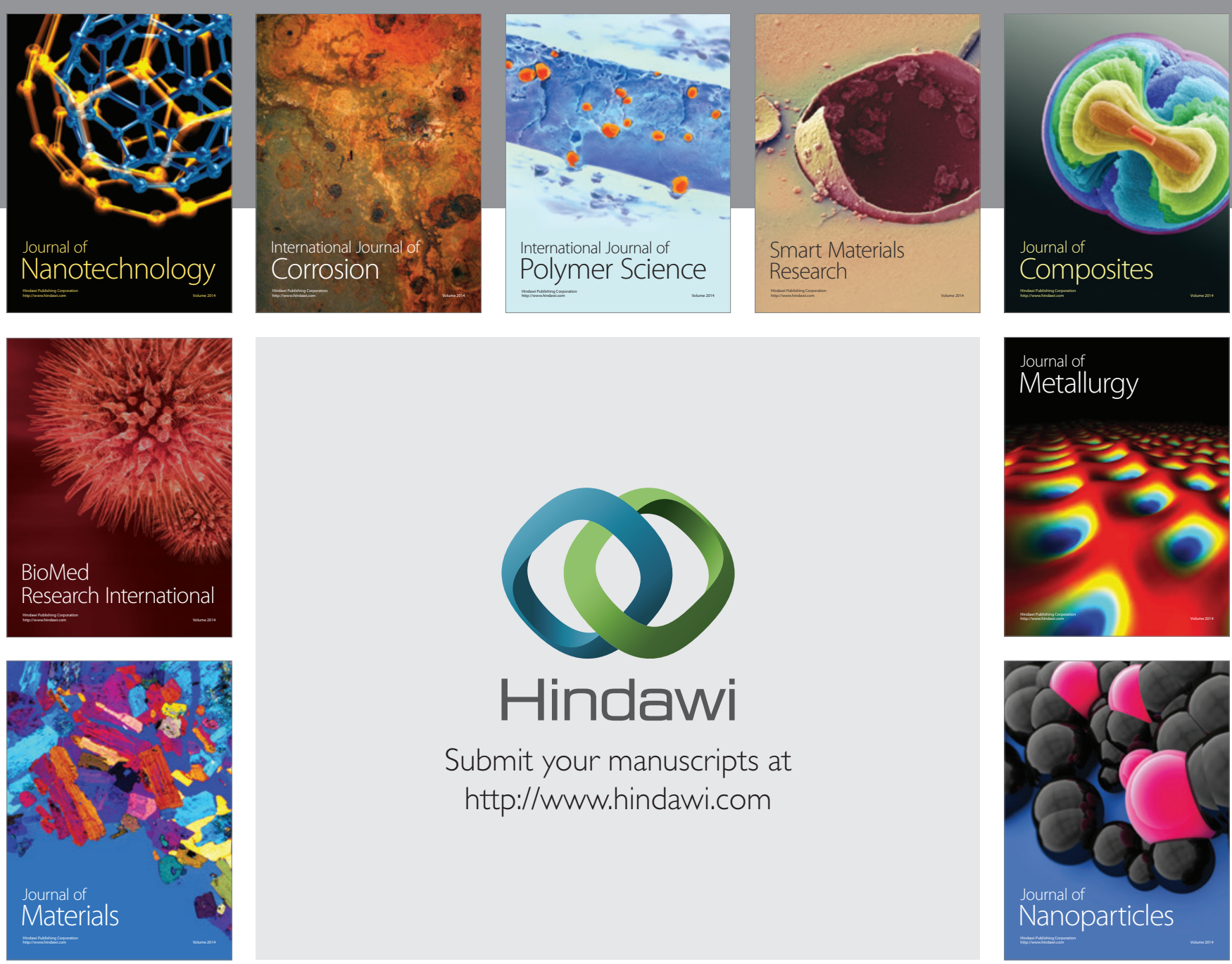

Submit your manuscripts at http://www.hindawi.com
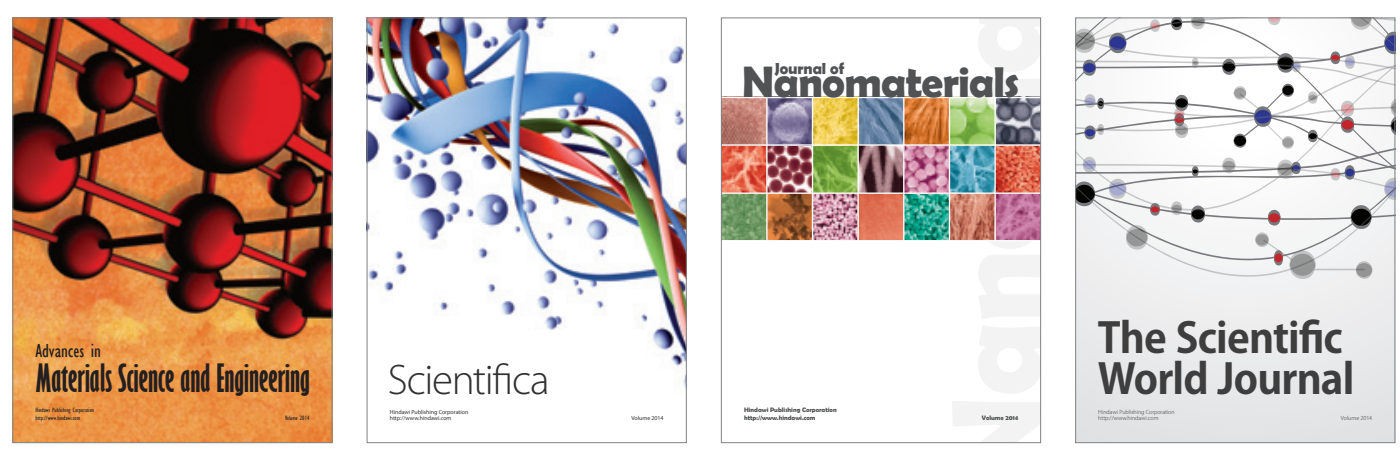

\section{The Scientific World Journal}
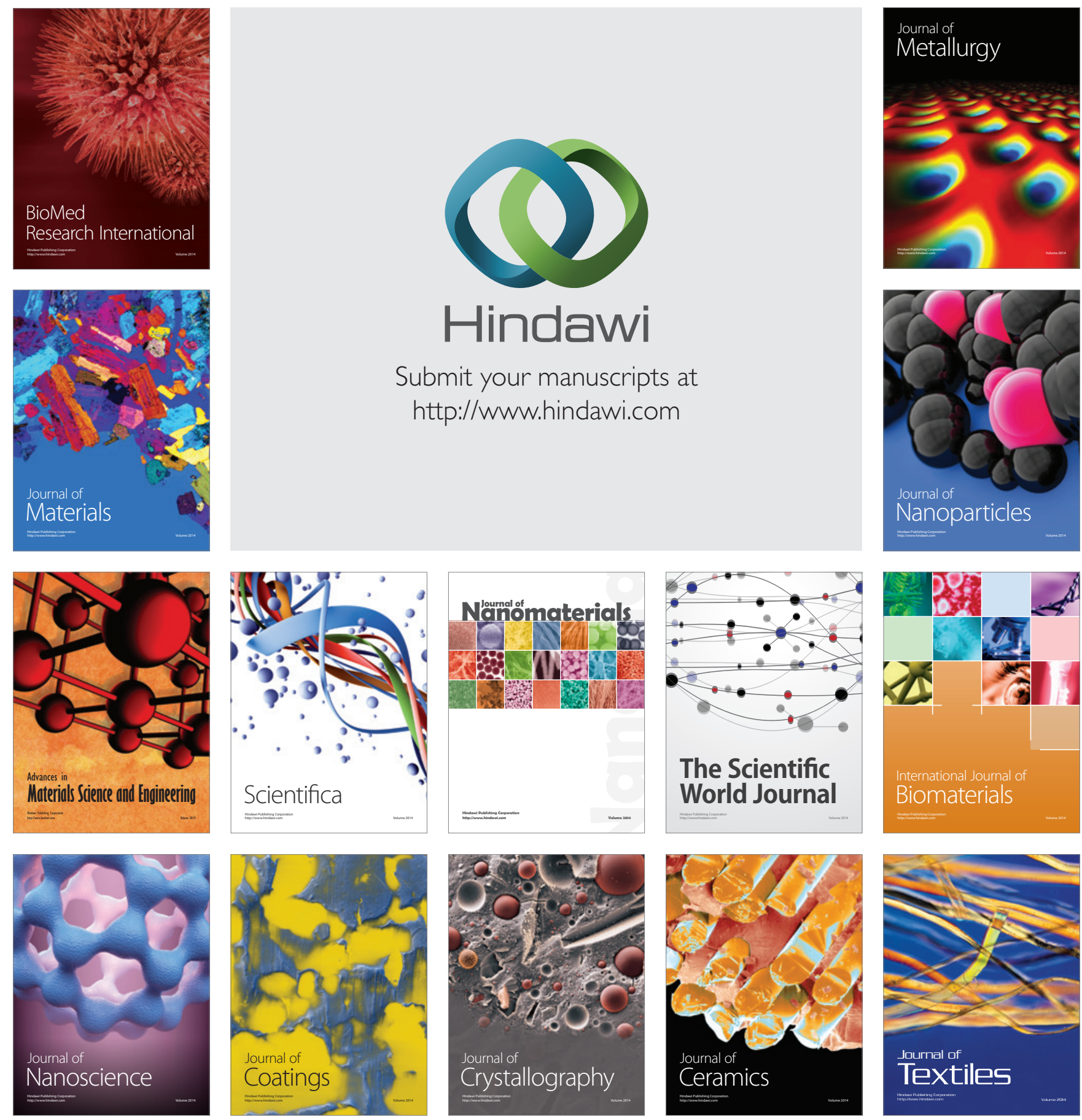